\title{
Topological quantum phase transition in the BEC-BCS crossover
}

\author{
Mitsuhiro Arikawa, ${ }^{1, *}$ Isao Maruyama, ${ }^{2}$ and Yasuhiro Hatsugai ${ }^{1}$ \\ ${ }^{1}$ Institute of Physics, University of Tsukuba, 1-1-1 Tennodai, Tsukuba, Ibaraki 305-8571, Japan \\ ${ }^{2}$ Graduate School of Engineering Science, Osaka University, 1-3 Machikaneyama-cho, Toyonaka, Osaka 560-8531, Japan
}

(Received 3 August 2010; published 30 August 2010)

\begin{abstract}
A crossover between the Bose-Einstein condensation (BEC) and BCS superconducting state is described topologically in the chiral symmetric fermion system with attractive interaction. Using a local $Z_{2}$ Berry phase, we found a quantum phase transition between the BEC and BCS phases without accompanying the bulk gap closing.
\end{abstract}

DOI: 10.1103/PhysRevB.82.073105

\section{INTRODUCTION}

Recent progress in ultracold atomic Fermi gases, the Feshbach resonance which controls strength and sign of effective interaction, realizes the Bose-Einstein condensation (BEC)-BCS crossover ${ }^{1-3}$ - Bose condensation of bosonic molecules which are realized in real-space pairing of fermions and the BCS superfluid with Cooper pairing in momentum space. A long time ago, Leggett ${ }^{4}$ proposed a trial wave function for the ground state which describes not only weakly attractive case where formation of the Cooper pairs occurs, but also the strong attractive case where a dilute gas of molecules undergoes the BEC. Also substantial number of theoretical works on the BEC-BCS crossover have been performed $^{5-7}$ after this pioneering work. By using a Ginzburg-Landau-type argument based on the symmetry breaking and local order parameter, these states are not clearly distinguished. They are considered as a crossover, that is, the two ground states are adiabatically connected even in the thermodynamic limit.

Both of the BCS and the BEC ground states are typical examples of gapped quantum states, which we call quantum liquids, in the sense that there are no symmetry-breaking phase transition between them. Still they are quite different and characteristic quantum states. As discussed in this Brief Report, there are clearly distinguished by using a topological quantity. Therefore they belong to a different class of matter as the topological insulator based on a classification scheme (topological classification).

A class of quantum Hall states is a typical example of topologically nontrivial quantum liquids where topological quantities play fundamental roles for the characterization. ${ }^{8-11}$ Also a time-reversal invariant analog of the quantum Hall states are studied intensively. ${ }^{12,13}$ As for the quantum liquids with nontrivial topological structure, it is proposed to use topological quantities such as the Berry phases and the Chern numbers using Berry connections. ${ }^{14-16}$ An important characteristic feature of the topological insulators is an appearance of local degrees of freedom near boundaries and impurities as generic edge states. ${ }^{8,11}$ Although the bulk is gapped and featureless, the edge states characterize the topologically nontrivial bulk. They are not independent but intimately related with each other, which is known as bulk-edge correspondence, where topologically nontrivial bulk guarantees the existence of localized modes and such low-energy localized excitations characterize the gapped bulk insulator ${ }^{11}$ conversely. The quantum Hall state of the graphene also belongs
PACS number(s): 64.70.Tg, 71.10.Fd, 73.20.-r, 73.43.-f

to this topological insulator where the bulk-edge correspondence is important for the description. ${ }^{18}$ The bulk-edge correspondence is also realized as the existence of the Kennedy triplet for an open integer chain. ${ }^{19}$ Such characteristic edge modes appear in the valence-bond solid $\operatorname{states}^{20}$ and spin ladder with cyclic-exchange interaction. ${ }^{17,21}$

In this Brief Report, we introduce a local $U(1)$ twist for the Hamiltonian and define a Berry phase using its manybody ground state, which is quantized into $Z_{2}$ due to the chiral symmetry of the Hamiltonian. ${ }^{14,22}$ Although the bulk gap is adiabatically connected between the BEC-BCS crossover, the gap of the twisted system may collapse at some value between the BEC-BCS crossover. It is allowed since the gap is not a thermodynamical property and can be collapsed by the local perturbation. It actually occurs in the BEC-BCS crossover. Then the crossover of the bulk is distinguished by the local quantum phase transition.

\section{MODEL AND $Z_{2}$ BERRY PHASE}

Let us start from the attractive Hubbard model at half filling and discuss a mean-field Hamiltonian

$$
\mathcal{H}=-t \sum_{\sigma=\uparrow, \downarrow} \sum_{\langle\mathbf{i}, \mathbf{j}\rangle} c_{\mathbf{i}, \sigma}^{\dagger} c_{\mathbf{j}, \sigma}-|U| \sum_{\mathbf{i}} n_{\mathbf{i}, \uparrow} n_{\mathbf{i}, \downarrow},
$$

where $c_{\mathbf{i}, \sigma}$ is annihilation operator at site $\mathbf{i}$ with spin $\sigma$. The number of sites is $N$. The summation over $\langle\mathbf{i}, \mathbf{j}\rangle$ is restricted to the nearest-neighbor pairs. The density with $\sigma$ spin at site $\mathbf{j}$ is given by $n_{\mathbf{j}, \sigma}=c_{\mathbf{j}, \sigma}^{\dagger} c_{\mathbf{j}, \sigma}$. We impose the periodic boundary condition. We assume the lattice is bipartite in one to three dimensions. To make the discussion clear, let us perform the following particle-hole transformation in the mean-field Hamiltonian $\mathcal{H}_{\mathrm{MF}}, u_{\mathbf{i}}=d_{\mathbf{i}, \uparrow}=c_{\mathbf{i}, \uparrow}$ and $d_{\mathbf{i}}=d_{\mathbf{i}, \downarrow}=c_{\mathbf{i}, \downarrow}^{\dagger}$ (see Fig. 1):

$$
\mathcal{H}_{\mathrm{MF}}=\sum_{\langle\mathbf{i}, \mathbf{j}\rangle}-t u_{\mathbf{i}}^{\dagger} u_{\mathbf{j}}+t d_{\mathbf{i}}^{\dagger} d_{\mathbf{j}}+\sum_{\mathbf{j}} \Delta\left(u_{\mathbf{j}}^{\dagger} d_{\mathbf{j}}+d_{\mathbf{j}}^{\dagger} u_{\mathbf{j}}\right) \text {. }
$$

The order parameter of the superconductivity $\Delta=$ $-|U|\left\langle c_{\mathbf{i}, \uparrow}^{\dagger} c_{\mathbf{i}, \downarrow}^{\dagger}\right\rangle=-|U|\left\langle u_{\mathbf{i}}^{\dagger} d_{\mathbf{i}}\right\rangle$ is chosen as a non-negative real number. In this Brief Report, we do not perform the selfconsistent calculation and just assume that the order parameter $\Delta$ is finite. Here the expectation values $\langle\mathcal{O}\rangle$ is taken for the ground state. The one-body eigenvalue problem of the $N$-site system is given by $\mathcal{H}_{\mathrm{MF}}\left|\phi^{(j)}\right\rangle=E^{(j)}\left|\phi^{(j)}\right\rangle\left(E^{(1)} \leq E^{(2)}\right.$ $\left.\leq \cdots \leq E^{(2 N)}\right)$, where the one-particle eigenstate is $\left|\phi^{(j)}\right\rangle$ $=\left(\phi^{(j)}\right)^{\dagger}|0\rangle=\left[\Sigma_{\mathbf{m}} \phi_{\mathbf{m}}^{(j, u)} u_{\mathbf{m}}^{\dagger}+\phi_{\mathbf{m}}^{(j, d)} d_{\mathbf{m}}^{\dagger}\right]|0\rangle$ with an orthogonal 


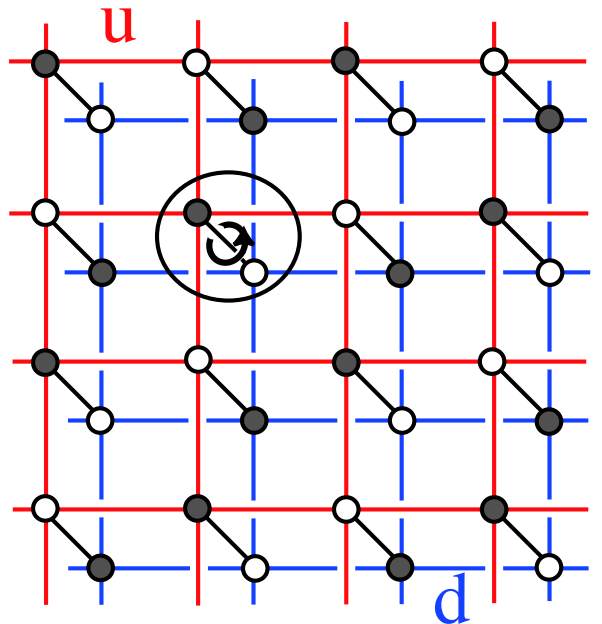

FIG. 1. (Color online) Lattice structure of the effective meanfield system for the two-dimensional case. We introduce a twist on the bond defined in Eq. (2).

normalization condition $\Sigma_{\mathbf{m}, \alpha}\left(\phi_{\mathbf{m}}^{(i, \alpha)}\right)^{*} \phi_{\mathbf{m}}^{(j, \alpha)}=\delta_{i j}$. Here the vacuum state $|0\rangle$ is defined as $u_{\mathbf{j}}|0\rangle=0=d_{\mathbf{j}}|0\rangle$ for any site $\mathbf{j}$. The $M$-particle eigenstate is constructed as $|\phi\rangle_{M}$ $=\prod_{j=1}^{M}\left(\phi^{(j)}\right)^{\dagger}|0\rangle$ with the eigenvalue $\sum_{j=1}^{M} E^{(j)}$. At half-filling band, by Fourier transform it is easily found that the ground state $|\phi\rangle_{N}$ has a finite excitation gap energy $2 \Delta$ for finite interaction $U$ for any bipartite system.

The BCS and BEC states are not clearly distinguished and they are adiabatically connected as a crossover even in the thermodynamic limit. In order to define the Berry phase, we modify the terms locally only at $\mathbf{j}=\mathbf{a}$ in Eq. (1) as follows:

$$
\Delta\left(u_{\mathbf{a}}^{\dagger} d_{\mathbf{a}}+d_{\mathbf{a}}^{\dagger} u_{\mathbf{a}}\right) \rightarrow \Delta\left(e^{i \theta} u_{\mathbf{a}}^{\dagger} d_{\mathbf{a}}+e^{-i \theta} d_{\mathbf{a}}^{\dagger} u_{\mathbf{a}}\right) .
$$

Hereafter we take $t$ as unity. Figure 2 presents the $\theta$ dependence of the energy spectrum of Eq. (3) for the square lattice. The energy gap $\delta E=E^{(N+1)}-E^{(N)}$ minimizes at $\theta=\pi$. In Fig. 2(b), we can see the level cross occurs at $(\theta, E)=(\pi, 0)$ around $\Delta=\Delta_{c} \simeq 1.250$ for two-dimensional case. As we will see later, $\Delta=\Delta_{c}$ is the critical point between the BEC and
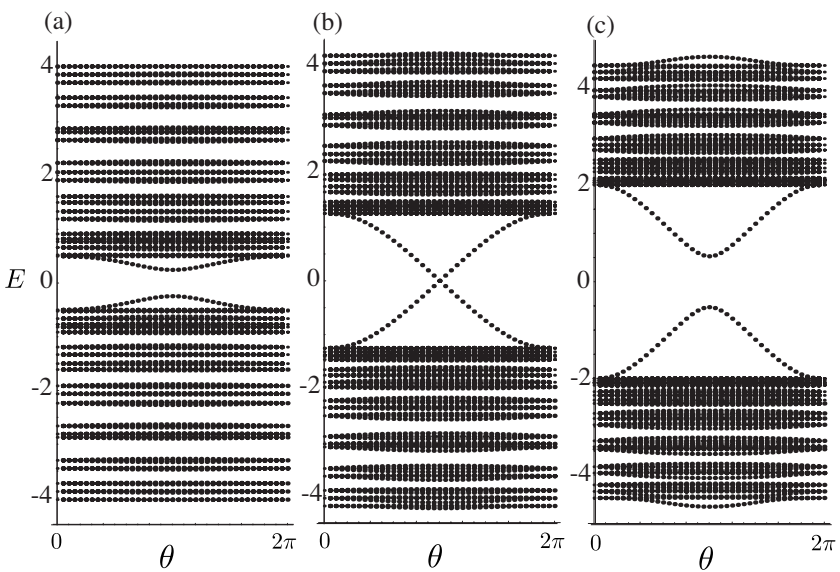

FIG. 2. Energy spectrum of $\mathcal{H}_{\mathrm{MF}}(\theta)$ for the two-dimensional case $(N=16 \times 16)$ : (a) $\Delta=0.5$. BCS phase. (b) $\Delta \simeq 1.250$. The level cross occurs at $(\theta, E)=(\pi, 0)$. (c) $\Delta=2$. BEC phase.
BCS phases of the BEC-BCS crossover, although the gap of the translational invariant system $(\theta=0)$ is always open.

Due to this local perturbation, the spectrum changes, which can be regarded as the edge mode. It characterizes the gapped bulk feature as a realization of the bulk-edge correspondence. Under the above deformation, the half-filled eigenstate $|\phi\rangle_{N}$ depends on the $\theta$, which denotes $|\phi(\theta)\rangle_{N}$. The Berry phase $\gamma$ is then defined as

$$
i \gamma=\int_{0}^{2 \pi} d \theta_{N}\left\langle\phi(\theta) \mid \partial_{\theta} \phi(\theta)\right\rangle_{N} .
$$

The transformation $\left(u_{\circ}, d_{0}\right) \rightarrow\left(-d_{0}, u_{\circ}\right)$ (see Fig. 1) for one sublattice reduces the Hamiltonian in the following bilinear form:

$$
\mathcal{H}_{\mathrm{MF}}(\theta)=\sum_{\mathbf{i}, \mathbf{j}} u_{\mathbf{i}}^{\dagger}\left[D_{N}(\theta)\right]_{\mathbf{i j}} d_{\mathbf{j}}+\text { H.c. }
$$

This Hamiltonian (3) has a chiral symmetry, $\left\{H_{\mathrm{MF}},{ }^{\exists} \gamma\right\}=0$, $\gamma^{2}=1$. Unless the determinant of the matrix $D_{N}(\theta)$ vanishes, the half-filling ground state of Hamiltonian (3) has a finite gap. Therefore, the Berry phase $\gamma$ is quantized as 0 or $\pi$ modulo $2 \pi\left(Z_{2}\right.$ Berry phase). ${ }^{14}$ Using this $Z_{2}$ Berry phase, we identify whether the half-filled ground state is a BEC or BCS state. The Berry phase Eq. (2) for the half-filled ground state of the chiral symmetric Hamiltonian (3) can be obtained as following expression: ${ }^{22}$

$$
\gamma=\int_{0}^{2 \pi} d \theta \operatorname{Im} \log \operatorname{det} D_{N}(\theta) .
$$

By definition, we can show that the $Z_{2}$ Berry phase remains invariant through an adiabatic deformation until the level cross occurs. That is, the Berry phase is topologically protected. In this Brief Report we evaluate the $Z_{2}$ Berry phase intuitively using an adiabatic continuation without direct calculation of Eq. (4).

\section{ADIABATIC DEFORMATION}

We consider the two types of the adiabatic deformation to understand the BCS and BEC states explicitly. Since we introduce the flux $\theta$ on one local bond, it turns out that $\operatorname{det} D_{N}(\theta)$ has a form $A e^{i \theta}+B$ ( $A$ and $B$ are real and independent of $\theta$ ) by the Laplace expansion. For a finite attraction $U$ (i.e., finite $\Delta$ ), only at $\theta=\pi$, det $D_{N}(\theta)$ can become zero where the level cross occurs at zero energy. During the change in the variable $\theta$ from 0 to $2 \pi$, the determinant det $D_{N}(\theta)$ draws a closed curve in the complex plane as shown in Fig. 3. If the det $D_{N}(\theta)$ winds around the origin of the complex plane $m$ times when $\theta$ varies from 0 to $2 \pi$, the Berry phase $\gamma$ is given by $\gamma=\pi m$ modulo $2 \pi$, if we assume Eq. (4). When the coupling strength satisfies that $0<\Delta$ $<\Delta_{c}$, the closed curve of $\operatorname{det} D_{N}(\theta)$ does not enclose the origin [see Fig. 3(a)]. From Eq. (4), we obtain the Berry phase $\gamma$ as $\gamma=0$. When $\Delta>\Delta_{c}$, the closed curve det $D_{N}(\theta)$ winds origin once [see Fig. 3(b)]. Then, we obtain $\gamma=\pi$. We do not need a proof for the general chiral Hamiltonian. We have justified Eq. (4) is valid using the adiabatic deformation 


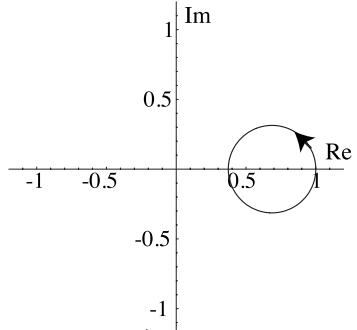

(a) $\Delta \triangleq 0.5$

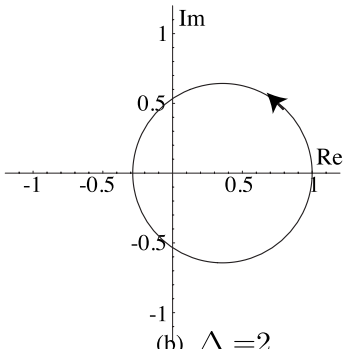

(b) $\Delta=2$
FIG. 3. The $\theta$ dependence of $\operatorname{det} D_{N}(\theta) / \operatorname{det} D_{N}(0)$ in the complex plane for $8 \times 8$ square lattice case. (a) $\Delta=0.5$ and (b) $\Delta=2$.

and the quantization of the Berry phase as we will discuss below.

Type (i). As seen in Fig. 3(a), for small $\Delta(=0.5)$, det $D_{N}(\theta)$ draws the circle without surrounding the origin in the complex plane. Then we adiabatically decrease the magnitude of the twist interlayer hopping $\Delta$ at $\mathbf{j}=\mathbf{a}$ to zero [see Fig. 4(a)]. During this adiabatic deformation, the circles in the complex plane shrink to the one point in a concentric fashion without touching the origin as the interlayer hopping $\Delta$ on the twist bond goes to zero [i.e., during the adiabatic deformation $A$ $\rightarrow 0$ and $B$ remains same when we write down $\operatorname{det} D_{N}(\theta)$ as $\left.\operatorname{det} D_{N}(\theta)=A e^{i \theta}+B\right]$. During this deformation, the excitation gap of the half-filling ground state remains finite. After the deformation, the dependence of $\theta$ disappears in Eq. (3). Due to the invariance of the $Z_{2}$ Berry phase under the adiabatic deformation, the Berry phase $\gamma$ should be zero for small $\Delta$, where the binding of the pairing is in momentum space (BCS state). In contrast, during the deformation, the circle touches the origin in the complex plane once for large $\Delta(=2.0)$. For large $\Delta$ the level cross occurs at zero energy for large $\Delta$. At the level-crossing point during the deformation, the Berry phase is not protected any more. To understand the Berry phase for large $\Delta$ we consider another type deformation.

Type (ii). We decrease the hopping $t$ connecting the site a into zero [see Fig. 4(b)]. Figure 3(b) shows the det $D_{N}(\theta)$ drawing the circle with surrounding the origin once in the complex plane. In this deformation, the circle is parallely shifted so that the center of the circle becomes the origin of the complex plane, i.e., $A$ remains same and $B \rightarrow 0$ for $\operatorname{det} D_{N}(\theta)=A e^{i \theta}+B$. During this deformation the level cross

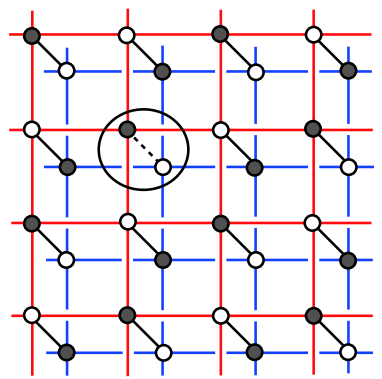

(a) BCS regime

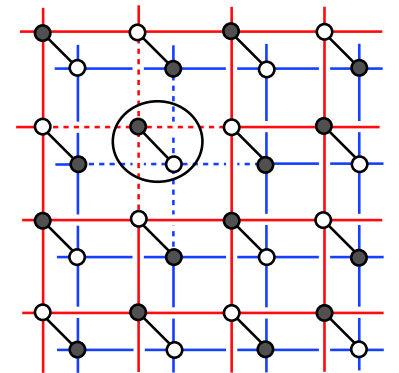

(b) BEC regime
FIG. 4. (Color online) Two types of the adiabatic deformations. One deformation is the decrease in the local bond (a) [type (i)]. The other is the decrease in the hopping connecting the deformed local bond being zero (b) [type (ii)].
TABLE I. Critical values $\Delta_{c}$ for dimensions $d=1,2$, and 3 .

\begin{tabular}{lc}
\hline \hline Dimension $d$ & $\Delta_{c}$ \\
\hline$d=1$ (linear chain) & $2 / \sqrt{3} \simeq 1.1547$ \\
$d=2$ (square lattice) & 1.250 \\
$d=3$ (cubic lattice) & 1.588 \\
\hline \hline
\end{tabular}

does not occur at zero energy for large $\Delta$. After the deformation, the twist interlayer hopping $\Delta$ at $\mathbf{j}=\mathbf{a}$ becomes isolated. Then, it is easy to show the isolated interlayer bond gives the $\pi$ Berry phase. Thus, the two electrons form a dimer bound state (BEC state) for large $\Delta$. When $\Delta$ is small, the Berry phase is zero as discussed above. During the deformation of type (ii), the circles touch the origin in the origin, that is, the level cross occurs at zero energy. To fix $\Delta_{c}$, we solve det $D_{N}(\pi)=0$ numerically for finite $N$. Then we perform the thermodynamic-limit extrapolation. From numerical calculation we find that there exists only one critical point $\Delta=\Delta_{c}$, where the Berry phase is $0(\pi)$ for $\Delta<\Delta_{c}\left(\Delta>\Delta_{c}\right)$. In a similar manner, the critical value $\Delta_{c}$ for the other dimensions $d$ can be calculated (see Table I). Near half filling the Berry phase $\gamma$ changes drastically from around zero to around $\pi$ when increasing the coupling $\Delta$, although it is crossover when the filling is not exactly one.

In one-dimensional case, the matrix $D_{N}(\theta)$ can be expressed as $\left[D_{N}(\theta)\right]_{i j}=\delta_{i-j, 0}\left[\Delta e^{i \theta} \delta_{i, 1}+(-1)^{i-1} \Delta\left(1-\delta_{i, 1}\right)\right]$ $+\left[\delta_{|i-j|, 1}+\delta_{|i-j|, N-1}\right] t$. The det $D_{N}(\theta)$ can be obtained analytically as

$$
\operatorname{det} D_{N}(\theta)=i \Delta e^{i \theta} U_{N-1}\left(\frac{i \Delta}{2}\right)-2 U_{N-2}\left(\frac{i \Delta}{2}\right)-2 .
$$

Here the $U_{n}(x)$ is the Chebyshev polynomial of the second kind, ${ }^{23}$ defined as $U_{n}(\cos x)=\sin [(n+1) x] / \sin x$. Parametrizing $\Delta$ by $\Delta=2 \sinh \alpha$, the asymptotic form of the $\operatorname{det} D_{N}(\theta)$ for large $N$ can be expressed as

$$
\operatorname{det} D_{N}(\theta) \simeq\left(i e^{\alpha}\right)^{N} \frac{e^{i \theta} \sinh \alpha+e^{-\alpha}}{\cosh \alpha} .
$$

We have a real root on $\alpha$ for the equation $\operatorname{det} D_{N}(\theta)=0$, i.e., $e^{i \theta} \sinh \alpha+e^{-\alpha}=0$ only when $\theta=\pi$. The solution is obtained as $\alpha=(\log 3) / 2$, i.e., $\Delta_{c}=2 / \sqrt{3} \simeq 1.1547$, although the bulk gap $(\theta=0)$ remains finite.

For one-dimensional system, the open boundary chain of Hubbard model is analyzed, ${ }^{24,25}$ which might be relevant to characterize the bulk features.

\section{SUMMARY AND DISCUSSIONS}

We show the quantum phase transition in the mean-field attractive Hubbard model at half filling. The $Z_{2}$ Berry phase distinguishes the BEC-BCS crossover as a local quantum phase transition, that is, the phases are separated by closing of the energy gap under the local twist, although the gap of the translational invariant system is always open. In the weak attractive interaction (BCS) case, the Berry phase is 0 in one hand. On the other hand, in the strong attraction (BEC) case, 


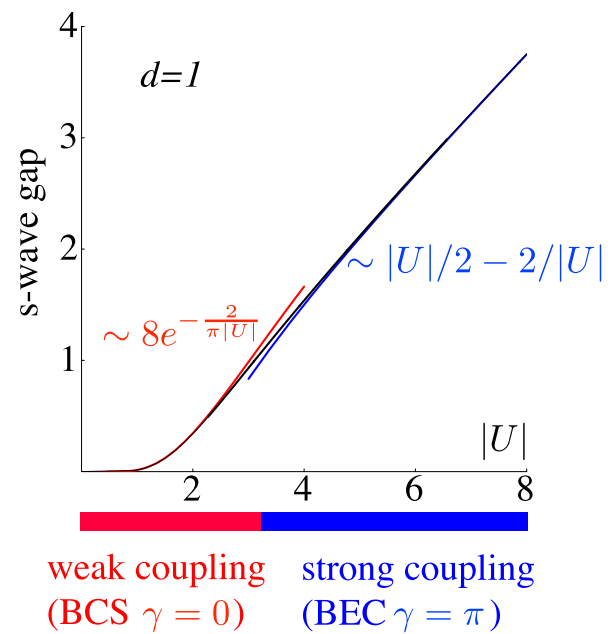

FIG. 5. (Color online) Relation between the $s$-wave gap $\Delta$ and the attractive interaction strength $|U|$ in one dimension. In weakcoupling (BCS) regime, $\Delta$ behaves $\Delta \sim 8 \exp -2 /(\pi|U|)$, where the Berry phase is 0 . In strong-coupling (BEC) regime, $\Delta \sim|U| / 2-2 /$ $|U|$, where the Berry phase is $\pi$. the Berry phase is $\pi$ (see Fig. 5). That is, it characterizes whether the paired electron is itinerant or localized. This comes from the bulk-edge correspondence in the BEC-BCS crossover. Physically the itinerant Cooper pairs in the BCS phase are not affected by the local $U(1)$ twist although the spatially localized bosons formed by the real space is crucially affected by the twist which results in the nontrivial $\pi$ Berry phase. In this sense, our topological characterization reflects whether the size of the pairing is macroscopic or of the order unity.

\section{ACKNOWLEDGMENTS}

The work was supported in part by Grants-in-Aid for Scientific Research, Grant No. 20340098 from JSPS and Grant No. 22014002 (Novel States of Matter Induced by Frustration) on Priority Areas from MEXT (Japan) (M.A., I.M., and Y.H.) and CREST in the Japan Science (M.A.). Some numerical calculations were carried out on Altix3700BX2 at YITP in Kyoto University and the facilities of the Supercomputer Center, Institute for Solid State Physics, University of Tokyo.
*Present address: Center for Computational Science, University of Tsukuba, Tsukuba, 305-8571, Japan and CREST, Japan Science and Technology Agency, 5 Sanbancho, Chiyoda, Tokyo 102-0075, Japan.

${ }^{1}$ C. A. Regal, M. Greiner, and D. S. Jin, Phys. Rev. Lett. 92, 040403 (2004).

${ }^{2}$ M. Bartenstein, A. Altmeyer, S. Riedl, S. Jochim, C. Chin, J. H. Denschlag, and R. Grimm, Phys. Rev. Lett. 92, 120401 (2004).

${ }^{3}$ M. W. Zwierlein, C. A. Stan, C. H. Schunck, S. M. F. Raupach, A. J. Kerman, and W. Ketterle, Phys. Rev. Lett. 92, 120403 (2004).

${ }^{4}$ A. J. Leggett, J. Phys. (Paris) 41, C7-19 (1980).

${ }^{5}$ P. Nozières and S. Schmitt-Rink, J. Low Temp. Phys. 59, 195 (1985).

${ }^{6} \mathrm{M}$. Randeria, in Bose-Einstein Condensation, edited by A. Griffin, D. W. Snoke, and S. Stringari (Cambridge University Press, Cambridge, 1995).

${ }^{7}$ Y. Ohashi and A. Griffin, Phys. Rev. Lett. 89, 130402 (2002).

${ }^{8}$ R. B. Laughlin, Phys. Rev. B 23, 5632 (1981).

${ }^{9}$ D. J. Thouless, M. Kohmoto, M. P. Nightingale, and M. den Nijs, Phys. Rev. Lett. 49, 405 (1982).

${ }^{10}$ X. G. Wen, Phys. Rev. B 40, 7387 (1989).

${ }^{11}$ Y. Hatsugai, Phys. Rev. Lett. 71, 3697 (1993).

${ }^{12}$ C. L. Kane and E. J. Mele, Phys. Rev. Lett. 95, 146802 (2005); 95, 226801 (2005).

${ }^{13}$ M. König, S. Wiedmann, C. Brüne, A. Roth, H. Buhmann, L. W.
Molenkamp, X.-L. Qi, and S.-C. Zhang, Science 318, 766 (2007).

${ }^{14}$ Y. Hatsugai, J. Phys. Soc. Jpn. 75, 123601 (2006).

${ }^{15}$ R. Resta, Berry's Phase and Geometric Quantum Distance: Macroscopic Polarization and Electron Localization, Lecture Notes for the "Troisième Cycle de la Physique en Suisse Romande" (Ecole Polytechnique Fédérale de Lausanne, Lausanne, 2000), also available online at http://www-dft.ts.infn.it/ resta/publ/ notes2000.ps

${ }^{16}$ T. Hirano, H. Katsura, and Y. Hatsugai, Phys. Rev. B 77, 094431 (2008); 78, 054431 (2008).

${ }^{17}$ I. Maruyama, T. Hirano, and Y. Hatsugai, Phys. Rev. B 79, 115107 (2009).

${ }^{18}$ M. Arikawa, Y. Hatsugai, and H. Aoki, Phys. Rev. B 78, 205401 (2008).

${ }^{19}$ T. Kennedy, J. Phys.: Condens. Matter 2, 5737 (1990).

${ }^{20}$ H. Katsura, T. Hirano, and Y. Hatsugai, Phys. Rev. B 76, 012401 (2007).

${ }^{21}$ M. Arikawa, S. Tanaya, I. Maruyama, and Y. Hatsugai, Phys. Rev. B 79, 205107 (2009).

${ }^{22}$ Y. Hatsugai and I. Maruyama (unpublished).

${ }^{23}$ T. S. Chihara, An Introduction to Orthogonal Polynomials (Gordon and Breach, New York, 1978).

${ }^{24}$ G. Bedürftig and H. Frahm, J. Phys. A 30, 4139 (1997).

${ }^{25}$ T. Deguchi, R. Yue, and K. Kusakabe, J. Phys. A 31, 7315 (1998). 